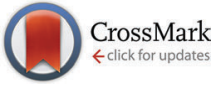

Cite this: Phys. Chem. Chem. Phys., 2016, 18, 3359

DOI: $10.1039 / c 5 c p 90229 k$

www.rsc.org/pccp

\section{Correction: The impact of doping rates on the morphologies of silver and gold nanowires grown in helium nanodroplets}

\author{
Alexander Volk, ${ }^{a}$ Philipp Thaler, ${ }^{a}$ Daniel Knez, ${ }^{b}$ Andreas W. Hauser, ${ }^{* a}$ \\ Johannes Steurer, ${ }^{a}$ Werner Grogger, ${ }^{b}$ Ferdinand Hofer ${ }^{b}$ and Wolfgang E. Ernst*a
}

Correction for 'The impact of doping rates on the morphologies of silver and gold nanowires grown in helium nanodroplets' by Alexander Volk et al., Phys. Chem. Chem. Phys., 2016, DOI: 10.1039/c5cp06248a.

Due to a unit conversion error, the authors would like to correct the values for $t_{\text {trap }}$ given in Table 2 of the published article, as seen in the amended Table below.

Table 2 Time $t_{\text {trap }}$ until particles get trapped by a vortex due to the attractive force given by eqn (6) and (7). $d_{D, \alpha}$ is calculated assuming an initial He $e_{N}$ size of $\bar{N}=1.7 \times 10^{10}$ atoms. $\bar{s}$ is calculated using eqn (8) and $d_{D, \alpha}$

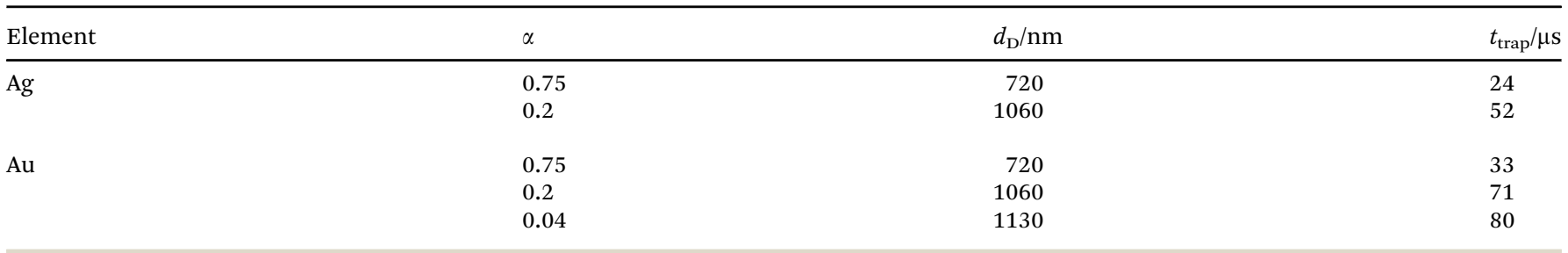

These changes of $t_{\text {trap }}$ affect some of the statements in the published article. Most notably, $t_{\text {trap }}$ is now shorter than the pickup process that takes place over the length of the pickup cell. The so-called pickup time just means that during $130 \mu \mathrm{s}$ new atoms are added. At first sight, one is tempted to assume a more complex growth process due to simultaneous effects of doping, cluster formation, and vortex trapping. However, this is not the case, since cluster formation times are still shorter than vortex trapping times $t_{\text {trap. }}$. In our cluster growth model following eqn (2) and (3) of the article, metal clusters of 100 atoms for light doping $(\alpha=0.2)$ and 1000 atoms for heavy doping $(\alpha=0.75)$ have formed already within $\approx 1 \mu$ s after pickup. The size distribution in Fig. 7 obtains its final shape very quickly and the changes between $30 \mu$ s (red curve) and $150 \mu$ s (orange curve) reflect only the subsequent growth due to atoms added over the $130 \mu$ s pickup time. Most of the 'newcomers' will become embedded into that stable cluster size distribution before they are getting trapped in a vortex (compare the envelopes at $130 \mu$ s (green curve) and $150 \mu \mathrm{s}$ in Fig. 7 of the main article). A slight shift of the maximum in the distribution functions towards larger particles with increasing time stems from the higher collision rates which occur due to the reduction of the droplet volume via He evaporation.

Due to this fact, the final morphology of most particles is still determined before they get captured by vortices. Our conclusions on the morphologies of the nanowires remain the same and are in agreement with the experimental results observed in the HRTEM pictures.

The Royal Society of Chemistry apologises for these errors and any consequent inconvenience to authors and readers.

\footnotetext{
${ }^{a}$ Institute of Experimental Physics, Graz University of Technology, Petersgasse 16, A-8010 Graz, Austria. E-mail: andreas.w.hauser@gmail.com, wolfgang.ernst@tugraz.at; Fax: +43 (316) 873 108140; Tel: +43 (316) 873 8157, +43 (316) 8738140

${ }^{b}$ Institute for Electron Microscopy and Nanoanalysis \& Graz Centre for Electron Microscopy, Graz University of Technology, Steyrergasse 17, A-8010 Graz, Austria
} 